\title{
Orientation polarization from faster motions in the ultraviscous and glassy diethyl phthalate and its entropy
}

\author{
G. Power and J. K. Vij ${ }^{\text {a) }}$ \\ Institute of Advanced Materials Science, Department of Electronic and Electrical Engineering, Trinity \\ College, University of Dublin, Dublin 2, Ireland \\ G. P. Johari \\ Department of Material Science and Engineering, McMaster University, Hamilton, \\ Ontario L8S 4L7, Canada
}

(Received 2 November 2005; accepted 29 November 2005; published online 27 January 2006)

\begin{abstract}
Dielectric spectra of the $\beta$ relaxation in glassy and ultraviscous liquid diethyl phthalate show that its relaxation strength $\Delta \epsilon_{\beta}$, the distribution of times, and the relaxation rate are more sensitive to temperature $T$ in the ultraviscous liquid than in the glassy state. The $\Delta \epsilon_{\beta}$ against temperature plot has an elbow-shaped break near $T_{g}$ of $\sim 181 \mathrm{~K}$, which is remarkably similar to that observed in the entropy, enthalpy, and volume against temperature plots, and in the plot of $\Delta \epsilon_{\beta}$ against the liquid's entropy minus its $0 \mathrm{~K}$ value. The ratio of $\Delta \epsilon_{\beta}$ to diethyl phthalate's entropy, after subtracting the $0 \mathrm{~K}$ value, is $1.08 \times 10^{-3} \mathrm{~mol} \mathrm{~K} / \mathrm{J}$ in the glassy state at $120.4 \mathrm{~K}$, which decreases slowly to 0.81 $\times 10^{-3} \mathrm{~mol} \mathrm{~K} / \mathrm{J}$ at $176 \mathrm{~K}$ near $T_{g}$ and thereafter rapidly increases to $1.57 \times 10^{-3} \mathrm{~mol} \mathrm{~K} / \mathrm{J}$ at $190 \mathrm{~K}$. Variation in $\Delta \epsilon_{\beta}$ parallels the variation of the entropy. A change in the activation energy of the $\beta$ process at $T>T_{g}$ indicates that its rate is also determined by the structure of the ultraviscous liquid. Features of $\beta$ relaxation are consistent with localized motions of molecules and may not involve small-angle motions of all molecules. () 2006 American Institute of Physics.
\end{abstract}

[DOI: $10.1063 / 1.2159473$ ]

\section{INTRODUCTION}

As part of our studies of the nature of the fast molecular motions in the glassy and ultraviscous liquids, we had found that the orientation polarization (or dielectric relaxation strength) due to these motions increased on heating, slowly in the glassy state until the glass-softening (transition) temperature $T_{g}$ was reached, and thereafter rapidly in the ultraviscous liquid. ${ }^{1}$ The temperature derivative of the relaxation strength therefore showed an abrupt increase over a narrow temperature range, which was similar in manner to that observed for the heat capacity $C_{p}$ and expansion coefficient in the temperature range near $T_{g}$. Because of this similarity between the dielectric and thermodynamic features, we intended to study the relaxation strength of a molecular glass whose heat capacity and entropy had been already measured. Chang and Bestul ${ }^{2}$ had reported such data for $o$-terphenyl and Chang et $a l^{3}$ have reported the data for diethyl phthalate ${ }^{3}$ in the glass, liquid, and crystal states. But, as the dipole moment of $o$-terphenyl is extremely small, the relaxation strength due to these fast motions in its glassy and ultraviscous liquid states is extremely small and could not be measured accurately enough to investigate the effect observed earlier. ${ }^{4}$ In contrast, diethyl phthalate has a relatively large dipole moment and an earlier study had shown that its dielectric loss peak is sufficiently high in the glassy state and therefore may allow comparison of the relaxation strength data with the $C_{p}$ and entropy data. When this work was being done, Pawlus et al. ${ }^{6}$ have reported the

${ }^{a)}$ Electronic mail: jvij@tcd.ie $\alpha$-relaxation spectra of diethyl phthalate over a broad temperature range in the ultraviscous liquid state and the secondary or faster relaxation spectra over a relatively narrow temperature range in the glassy and ultraviscous liquid state. They also studied the effect of pressure on both relaxations. Based on these studies, Pawlus et al. ${ }^{6}$ and Ngai and Paluch ${ }^{7}$ deduced that the sub- $T_{g}$ relaxation peak in diethyl phthalate may not be the true Johari-Goldstein $(\mathrm{JG})$ relaxation, but may have an intramolecular origin. Their reasoning is based principally on an experimental requirement that at two pressure-temperature states where the $\alpha$ relaxation is superposable, $f_{m, \beta}$ is the same, ${ }^{6}$ and the sub- $T_{g}$ relaxation of diethyl phthalate failed this requirement. Accordingly, we refer this secondary relaxation as the $\beta$ relaxation here, and maintain that any intramolecular motion of segments of a molecule would still occur in an intermolecular environment, i.e., the barrier for the segmental motions may still be determined by intermolecular interaction. Ngai and Paluch ${ }^{7}$ have reviewed this subject recently. In the study of Pawlus et al. ${ }^{6}$ the relaxation strength of the $\beta$ process was not measured. Also the $\beta$-relaxation rate of the ultraviscous liquid was reported at only a few temperatures above $T_{g}$. Here we report the relaxation strength and extended relaxation rate data at more temperatures and over a wider range from accurate and rapid dielectric spectra measurements.

Consequences of the $\beta$ relaxation have been seen also in calorimetric studies. Accurate $C_{p}$ measurements of several molecular glasses have shown a weak and broad endothermic features at a temperature where the extrapolation of the rate of the $\beta$-relaxation process is of the order of $1 \mathrm{MHz}^{8-10}$ 
This feature has been therefore attributed to the gradual, kinetic unfreezing of the $\beta$ relaxation on heating and by analogy with $T_{g}$ at which the $\alpha$-relaxation process kinetically unfreezes gradually on heating, the onset temperature of this $C_{p}$ endotherm has been referred to as $T_{g, \beta}$. More recently, a fictive temperature for the JG relaxation has been defined ${ }^{11}$ by using James Prescott Joule's 1884 data on his glass thermometer's zero-point shift observed over a period of about 39 years. $^{12}$ (It should be noted that Joule's observations on aging of a silica-based glass stored at ambient temperature has been verified by others, as described earlier, ${ }^{11}$ and his observations have finally led to development of glass compositions for construction of more reliable thermometers in the 19th and 20th centuries.) Thus there is independent evidence for contributions from the JG relaxation to the changes in the calorimetric properties and volume of a glass on aging.

Goldstein $^{13,14}$ and Johari ${ }^{15-17}$ had used the available calorimetric data to examine the source of the excess heat capacity $C_{p \text {,exc }}$ and excess entropy $S_{\text {exc }}$ of a liquid and a glass over the crystal state. They found that the $C_{p, \text { exc }}$ and $S_{\text {exc }}$ of the ultraviscous liquid state are due to the change in the vibrational frequencies with change in the configurational state, the anharmonic (vibrational) displacement, the molecular rearrangement process that appears as a secondary relaxation, and the unfrozen modes of motions in the broad distribution of the $\alpha$-relaxation process. In the glassy state, $C_{p \text {,exc }}$ and $S_{\text {exc }}$ were seen to be partly due to the difference between the vibrational frequencies of the glass and crystal, partly due to anharmonic (vibrational) displacement and partly from the localized molecular motions in the disordered structure of a glass. ${ }^{13-16}$ A more recent examination ${ }^{17}$ has suggested that there is a further configurational contribution from the faster modes of motions of the $\alpha$-relaxation process that remain kinetically unfrozen at $T<T_{g}$. Goldstein ${ }^{14}$ had examined also the variation of $C_{p \text { exc }}$ and $S_{\text {exc }}$ of diethyl phthalate glass with temperature from its $T_{g}$ to near $0 \mathrm{~K}$, and found that the decrease in its $S_{\text {exc }}$ on cooling from $T_{g}$ to near $0 \mathrm{~K}$ is much smaller than for other glasses, meaning that there is only a minor contribution to its $C_{p \text {,exc }}$ and $S_{\text {exc }}$ from factors other than the configurational states. Diethyl phthalate appeared to be anomalous amongst the six substances whose $C_{p \text { exc }}$ and $S_{\text {exc }}$ data he had analyzed. ${ }^{14}$ A previous study had already shown a $\beta$-relaxation peak in its glassy state. ${ }^{5}$

One of the characteristic features of the $\beta$ relaxation is that its relaxation strength increases with increase in the temperature $T .{ }^{4,5,18}$ This means that as the number of molecules contributing to the availability of distinct configurations increases with increase in $T, C_{p \text {,exc }}$ and $S_{\text {exc }}$ and any contribution to volume should increase, and hence one would expect that the increase in the $\beta$-relaxation strength may be at least qualitatively related to $C_{p \text { exc }}$ and $S_{\text {exc }}$ and possibly to free volume. Bartoš and co-workers ${ }^{19-25}$ have studied a series of glasses, both molecular and polymeric and have found that as $T$ is increased, the hole volume $V_{h}$ in the structures of the glass and ultraviscous liquid also increases slowly. It increases slowly in the glassy state and increases rapidly in the ultraviscous liquid or rubber state at $T>T_{g}$, thus showing an elbowlike feature in its plot against $T$. Thus, two more quan- tities, namely, $\left(d \Delta \epsilon_{\beta} / d T\right)_{p}$ [see Ref. 1] and $\left(d V_{h} / d T\right)_{p}$ [Refs. 19-25] have been recently found to change with change in $T$ in the same manner as $C_{p}$ changes when configurational freezing occurs on cooling and unfreezing occurs on heating through the $T_{g}$ range. Here we report a dielectric relaxation study of the $\beta$-relaxation process and investigate whether: (i) the $\beta$ relaxation's strength and rate have a structuredependent contribution, (ii) the variation in the relaxation strength and rate can be qualitatively related to the entropy, and (iii) the suggestion for the origin of $\beta$ relaxation in terms of small-angle motions of all molecules in a glass can be verified. Pawlus et al. ${ }^{6}$ have already provided exhaustive data on the $\alpha$-relaxation process in diethyl phthalate and our data agree with theirs. Therefore, we report only the features of the $\beta$-relaxation spectra from our detailed study of diethyl phthalate. The remainder of the data may be found in Ref. 26.

\section{EXPERIMENT}

Diethyl phthalate (99.5\% pure) was purchased from Fluka and used in its liquid state. The dielectric cell was a silver-plated capacitor with 18 plates immersed in a small vial containing the liquid at room temperature together with a $100 \Omega$ platinum resistor used as a temperature sensor. The cell was placed in a cryostat cooled by a nitrogen gas jet system with a Novocontrol Quatro temperature controller. The nitrogen gas also provides an inert atmosphere around the sample. The maximum cooling rate of the system was used so that the sample cooling rate slowed as the base temperature of $119.5 \mathrm{~K}$ was approached in $\sim 1.3 \mathrm{~h}$. The sample was then heated to $226.3 \mathrm{~K}$ at $4.9 \mathrm{~K} / \mathrm{h}$ rate. During heating the dielectric permittivity $\epsilon^{\prime}$ and loss $\epsilon^{\prime \prime}$ spectra in the frequency range of $1 \mathrm{~Hz}-10 \mathrm{MHz}$ were measured continuously using a Novocontrol Alpha broadband dielectric spectrometer. The measurement of one spectrum took $135 \mathrm{~s}$, during which time the temperature changed by about $0.18 \mathrm{~K}$, giving an uncertainty in the mean temperature of $\pm 0.09 \mathrm{~K}$.

\section{RESULTS}

The permittivity $\epsilon^{\prime}$ and loss $\epsilon^{\prime \prime}$ spectra at several temperatures below the adiabatic calorimetric $T_{g}$ of $\sim 176-181 \mathrm{~K}$ (Ref. 2) are shown in Figs. 1(A) and 1(B), and those at higher temperatures are shown in Figs. 2(A) and 2(B). An $\epsilon^{\prime \prime}$ peak is seen clearly in the spectra at low temperatures. As the temperature is increased, the $\epsilon^{\prime \prime}$ peak height increases. Despite that the peak becomes progressively less resolved because of the increase in the proximity of the $\alpha$-relaxation peak to the $\beta$-relaxation peak, and hence the progressively increasing contribution from the $\alpha$ relaxation to the $\epsilon^{\prime}$ and $\epsilon^{\prime \prime}$ values in the frequency range where the $\beta$-relaxation loss peak is expected. This feature of ultraviscous liquids has been noted since 1970 . Thus only a highfrequency shoulder appears at $201.1 \mathrm{~K}$. Only the $\epsilon^{\prime \prime}$ peak due to the $\alpha$ relaxation is observed at high temperature in Fig. 2(A). There is an inverted sigmoid shape decrease in $\epsilon^{\prime}$ corresponding to the $\alpha$ and $\beta$ peaks, which is seen in Figs. 1(A) and 2(A). 


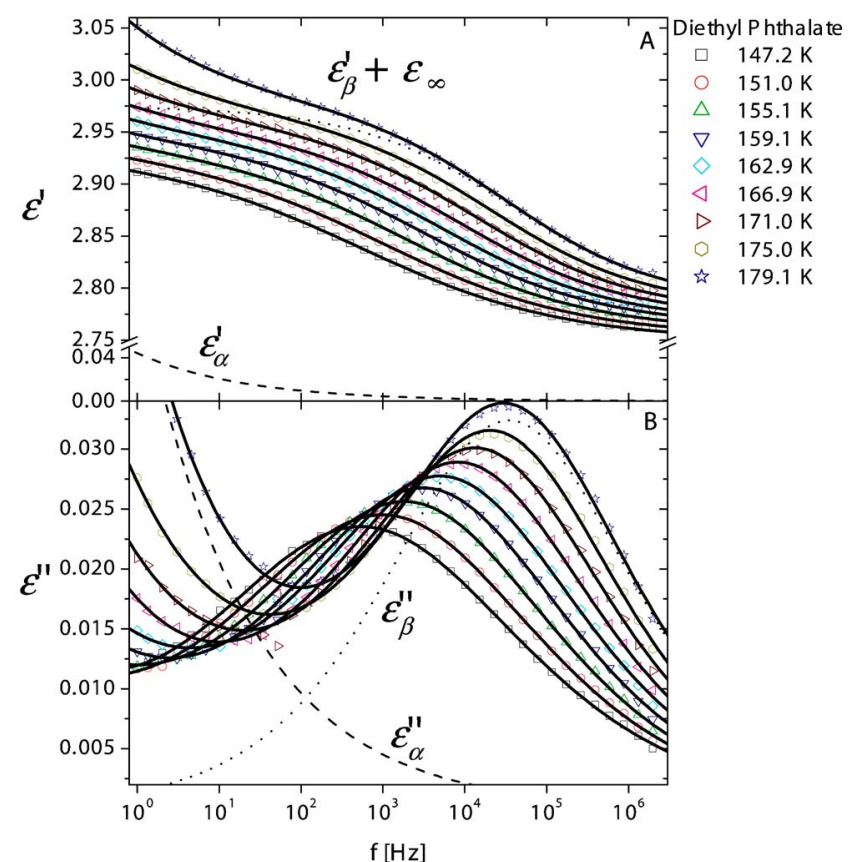

FIG. 1. (Color online) (A). The $\epsilon^{\prime}$ spectra of diethyl phthalate at several temperatures below $T_{g}$. (B) The corresponding $\epsilon^{\prime \prime}$ spectra. Solid lines through the data in both panels are fits of Eq. (1) to the data. The decomposition of the fit into its various terms is shown for $179.1 \mathrm{~K}$. The $\alpha$-relaxation contribution is shown as a dashed line, and the $\beta$ relaxation is shown as a dotted line.

To determine the contribution to dielectric relaxation strength, the relaxation time distribution parameter, and the frequency of maximum loss $f_{m, \beta}$ of the $\beta$-relaxation process, the empirical Havriliak-Negami (HN) equation ${ }^{27}$ was fitted to the data as described previously ${ }^{1,28-30}$ using the winfIT dielectric fitting program. Since the $\epsilon^{\prime \prime}$ spectra contains lowand a high-frequency loss peaks from the $\alpha$ and $\beta$ relaxations, we use an equation containing terms for both relaxations and an imaginary term proportional to the dc conductivity $\sigma_{d c}$ for the loss from ionic conduction. The complex permittivity $\epsilon^{*}(\omega)$, where $\omega=2 \pi f(f$ in $\mathrm{Hz})$, is given by

$$
\epsilon^{*}(\omega)=\epsilon_{\infty}+\frac{\Delta \epsilon_{\alpha}}{\left[1+\left(j \omega \tau_{\alpha}\right)^{\alpha}\right]^{\beta_{\alpha}}}+\frac{\Delta \epsilon_{\beta}}{\left[1+\left(j \omega \tau_{\beta}\right)^{\alpha_{\beta}}\right]^{\beta_{\beta}}}-j \frac{\sigma_{\mathrm{dc}}}{\epsilon_{0} \omega} .
$$

$\epsilon_{\infty}$ is the limiting permittivity at high frequencies, which depends on atomic and electronic polarizibility. $\Delta \epsilon_{\alpha}$ is the dielectric strength, $\tau_{\alpha}$ the characteristic relaxation time, $\alpha_{\alpha}$ the symmetric broadness parameter (this is equivalent to 1 $-\alpha_{\mathrm{CC}}$, where $\alpha_{\mathrm{CC}}$ is the Cole-Cole distribution parameter ${ }^{31}$ ), and $\beta_{\alpha}$ is the asymmetric broadness parameter (equivalent to the Davidson-Cole parameter ${ }^{32}$ ) of the $\alpha$ relaxation; $\Delta \epsilon_{\beta}, \tau_{\beta}$, $\alpha_{\beta}$, and $\beta_{\alpha}$ are the corresponding parameters for the $\beta$ relaxation. The imaginary part of Eq. (1) is fitted to $\epsilon^{\prime \prime}$, and the real part adjusted to obtain $\epsilon_{\infty}$.

The data were analyzed as follows. For spectra at $140 \mathrm{~K}$ and lower $T$ only the $\beta$-relaxation term and a small, unanalyzable relaxation term at high frequencies were fitted to the data. The best fits of the spectra were obtained when $\beta_{\beta}=1$, showing that the $\beta$-relaxation spectra have a symmetric or Cole-Cole distribution. ${ }^{31}$ For all subsequent fits, $\beta_{\beta}=1$ was

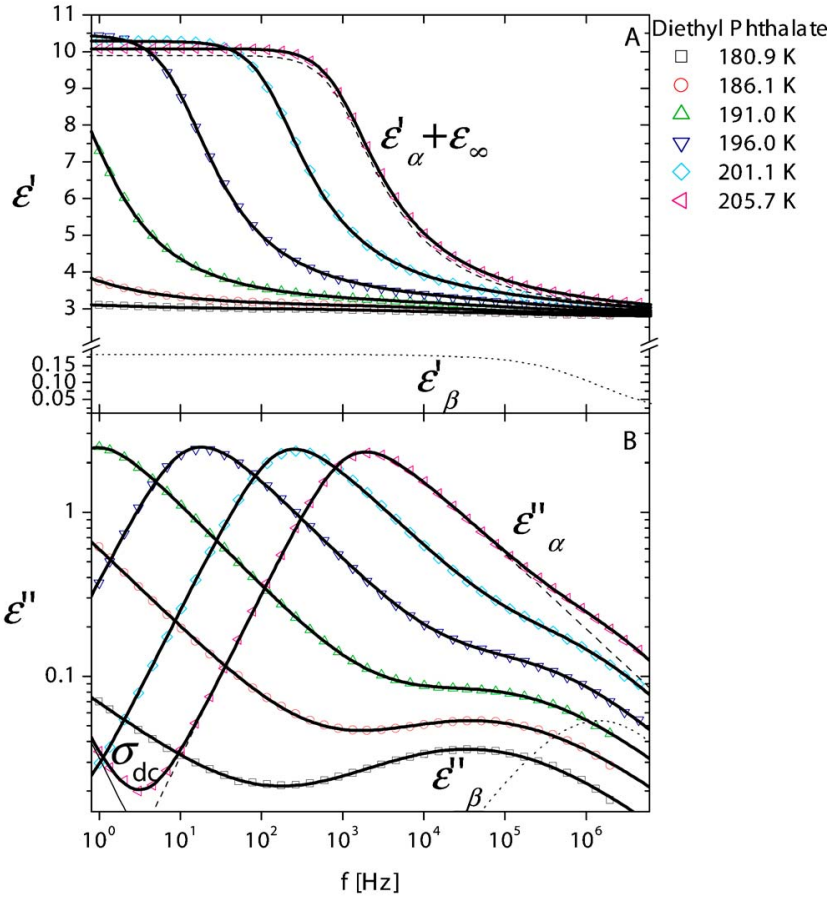

FIG. 2. (Color online) (A) The $\epsilon^{\prime}$ spectra of diethyl phthalate at several temperatures above $T_{g}$. (B) The corresponding $\epsilon^{\prime \prime}$ spectra. Solid lines through the data in both panels are fits of Eq. (1) to the data. The decomposition of the fit is shown for $205.7 \mathrm{~K}$. The $\alpha$-relaxation contribution is shown as a dashed line, and the $\beta$ relaxation is shown as a dotted line. The dc conductivity contribution to $\epsilon^{\prime \prime}$ is shown as a thin solid line.

used. Also, for $T>140 \mathrm{~K}$, the $\alpha$-relaxation term was included. As the $\alpha$ relaxation could not be characterized fully at these low temperatures, its contribution was estimated by assuming a Davidson-Cole relaxation process with $\beta_{\alpha}=0.5$. $\Delta \epsilon_{\alpha}$ was estimated by a linear extrapolation of the values at high temperature to the measurement temperature, and only $\tau_{\alpha}$ was fitted as a free parameter (with values in the megasecond range). At $T>149 \mathrm{~K}, \beta_{\alpha}$ was also fitted as a free parameter, because a fixed $\beta_{\alpha}=0.5$ gave poor fits as $T$ was increased. At $T>174 \mathrm{~K}$, the $\alpha$-relaxation contribution to $\epsilon^{\prime \prime}$ became appreciable, and therefore a logarithmic scale $\epsilon^{\prime \prime}$ fit was used instead of a linear scale. At $T>189 \mathrm{~K}, \Delta \epsilon_{\alpha}$ was fitted as a free parameter. At $T>191 \mathrm{~K}, \alpha_{\alpha}$ was also fitted as a free parameter, as required. The contribution from the $\mathrm{dc}$ conductivity was added at $T>200 \mathrm{~K}$. At $T>209 \mathrm{~K}$, the $\beta$-relaxation term was removed from the fitting equation since its contribution became negligible - only the $\alpha$ relaxation and $\sigma_{\mathrm{dc}}$ terms were used for fitting. Reliable $\beta$-relaxation parameter values are obtained for 120.4-195.4 K. At the ultimate temperature of $195.4 \mathrm{~K}$, the $\beta$-relaxation loss peak is still approximately 1.56 decades (corresponding to eight data points) away from the $\epsilon^{\prime \prime}$ data point of highest frequency included in the fit and was still visible as a shoulder on the larger $\alpha$ relaxation [see Fig. 2(B)]. Parameters obtained for the $\alpha$ relaxation at $T$ $<192.1 \mathrm{~K}$ are not reliable because the peak is not observed in the spectra. The frequency of maximum loss $f_{m}$ of each relaxation process is found from ${ }^{33}$ 


$$
f_{m}=(2 \pi \tau)^{-1}\left[\sin \left(\frac{\alpha \pi}{2+2 \beta}\right)\right]^{1 / \alpha}\left[\sin \left(\frac{\alpha \beta \pi}{2+2 \beta}\right)\right]^{-1 / \alpha} .
$$

For $\beta=1$ we obtain using Eq. (2) $f_{m}=(2 \pi \tau)^{-1} \cdot f_{m}$ is therefore found to be independent of $\alpha$. The solid lines in Figs. 1 and 2 are the plots of Eq. (1) constructed by using the parameters obtained in fitting. The total fit of the spectra was done as sum of the contribution from the $\alpha$ - and $\beta$-relaxation terms in Eq. (1). The best fits of the spectra were obtained when $\beta_{\beta}=1$, showing that the $\beta$-relaxation spectra have a symmetric or Cole-Cole distribution.

There are several possible sources of error in the measurement. First, there is a systematic error in the fitting parameters because the temperature rises at a constant rate during the scan. This has been estimated by assuming that the trends in the temperature dependence of the $\beta$-relaxation parameters can be obtained from the results. For $\alpha_{\beta}$ and $\Delta \epsilon_{\beta}$ in the glass the uncertainty is $<0.1 \%$ and falls with temperature because both parameters increase while the absolute uncertainty is constant. For $\alpha_{\beta}$ and $\Delta \epsilon_{\beta}$ above the transformation region, it is $0.2 \%$ and $1 \%$, respectively. The uncertainty in $\log \left(f_{m, \beta}\right)$ is about $2 \%$ at $120.4 \mathrm{~K}$, which is 0.01 decades. It falls as the temperature increases below $T_{g}$ and is $<0.1 \%$ at $180 \mathrm{~K}\left(<0.01\right.$ decades). Above $T_{g}$ it starts to increase, but is still less than $0.2 \%$ ( 0.01 decades). Second, the absolute error in the measured temperature is of the same order as the temperature uncertainty due to the ramp $(0.1 \mathrm{~K})$ so it should contribute similarly to the uncertainty in the fit parameters. Third, there is an uncertainty in the parameters as a result of the fitting, which has been estimated by the WINFIT program. Uncertainty in $\alpha_{\beta}$ and $\Delta \epsilon_{\beta}$ arising from the data analysis itself is not more than $1 \%$ for all temperatures. It is highest at low temperatures and rises again in the region where the $\alpha$ and $\beta$ processes begin to overlap. The highest uncertainty in $\log \left(f_{m, \beta}\right)$ due to fitting is about $5 \%$ at $120.4 \mathrm{~K}$, where the relaxation is very broad. Above this, the uncertainty in $\log \left(f_{m, \beta}\right)$ due to data analysis is not more than $0.4 \%$. The third source of uncertainty in the parameters is the measuring equipment itself. Using Novocontrol's absolute error estimates for measurements with the alpha analyzer and assuming an uncertainty in the empty cell capacitance of $\sim 1 \%$, the worst points in the $\epsilon^{\prime}$-spectra (at high frequencies $>1 \mathrm{MHz}$ ) have an uncertainty of $\sim 2 \%$.

Using the simplifications that the uncertainties in the measurement have a similar effect on the fitting parameters, and they are additive, the uncertainties in the $\alpha_{\beta}, \Delta \epsilon_{\beta}$, and $\log \left(f_{m, \beta}\right)$ values around the region where the $\alpha$ and $\beta$ relaxations start to overlap (195.4 K) are roughly estimated to be $3 \%, 5 \%$, and $3 \%$, respectively.

The values of $\Delta \epsilon_{\beta}$ and $\alpha_{\beta}$ obtained from this analysis are plotted against the temperature, respectively, in Figs. 3(A) and 3(B). $\Delta \epsilon_{\beta}$ increases slowly with increase in $T$ from 0.17 at $120 \mathrm{~K}$ to 0.19 at $178 \mathrm{~K}$. At $T>T_{g}$, it increases more rapidly, i.e., there is a change of slope in the $\Delta \epsilon_{\beta}$ against $T$ plot in the glass-softening range, giving the plot an elbow shape. At temperatures higher than $190 \mathrm{~K}$ the plot seems to curve slightly downwards. This is due to crystallization of diethyl phthalate on heating. The elbow shape is similar to that observed generally for the volume, enthalpy, and entropy plots

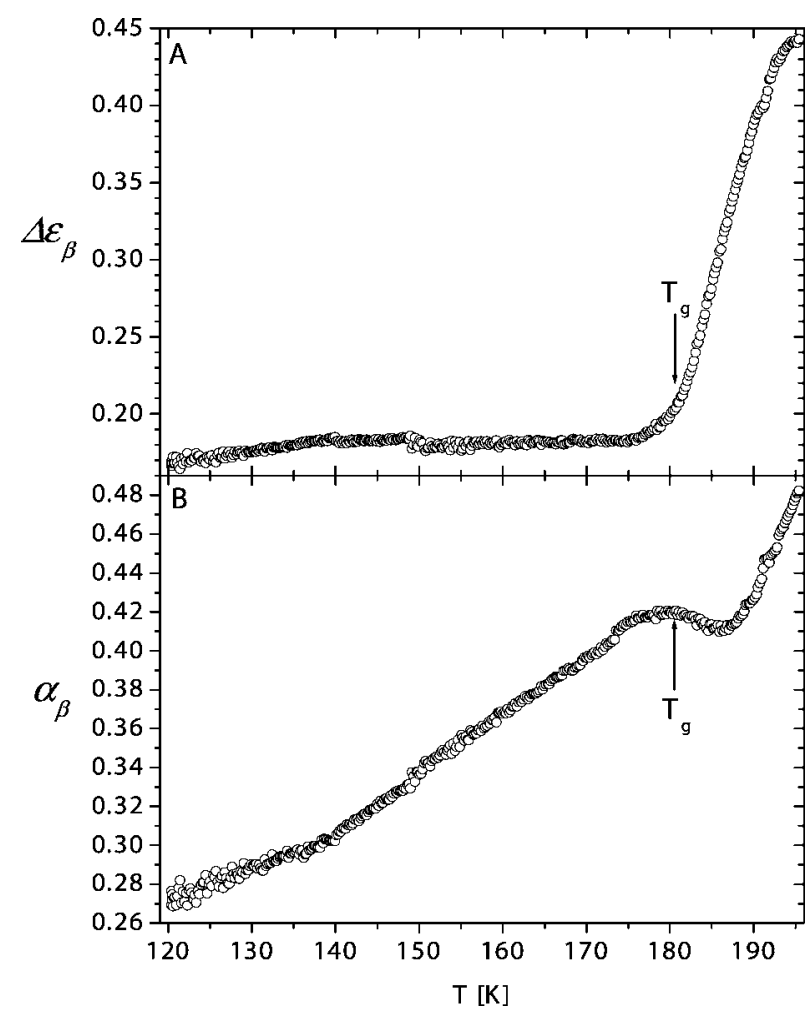

FIG. 3. (A) Plots of the resolved $\Delta \epsilon_{\beta}$ for diethyl phthalate against the temperature. (B) The corresponding plot of the distribution parameter $\alpha_{\beta}$ against the temperature.

and is seen as a characteristic of the glassy state. More recently the same elbow shape has been observed in the corresponding plot of the hole volume measured by positron annihilation lifetime spectroscopy (PALS). ${ }^{20-25}$ The plot of $\alpha_{\beta}$ against the temperature in Fig. 3(B) shows a shallow minimum at $T$ immediately above $T_{g}$ in the $180-187 \mathrm{~K}$ range. This seems to be beyond the analytical errors of the WINFIT fitting program discussed above.

Figure 4 shows the logarithmic plots of $f_{m, \alpha}$ and $f_{m, \beta}$ against $1 / T$. Our data for $f_{m, \alpha}$ and $f_{m, \beta}$ agree with the data of Pawlus et al. ${ }^{6}$ in the temperature range of the overlap. However, the $\log \left(f_{m, \beta}\right)$ against $1 / T$ plot shows a relatively small, constant slope in the glassy state, with a low Arrhenius energy and the data fit to the relation, $f_{m}=10^{13} \exp (-28797 / R T)$. As the temperature is increased from the $T_{g}$ range, the slope of the $\log \left(f_{m, \beta}\right)$ against $1 / T$ plot first decreases slightly and then increases. The slope increases in the glass-softening range and remains high for the ultraviscous liquid state at $T>T_{g}$, where it remains unchanged with time.

It should be stressed that the unusual inflexion in the $\alpha_{\beta}$, against $T$ plot in Fig. 3(B) appears at a temperature where $f_{m, \alpha}$ is at least two orders of magnitude lower than $f_{m, \beta}$, indicating that the two relaxations are well separated and should be reliably resolvable, despite the fact that we need one parameter, $\tau_{\beta}$, for determining $f_{m, \beta}$, and three parameters, $\tau_{\alpha}, \alpha_{\alpha}$, and $\beta_{\alpha}$ for determining $f_{m, \alpha}$. Although computational fits seek the best parameters, an ambiguity that arises from experimental errors and errors in determining $\tau_{\alpha}, \alpha_{\alpha}$, and $\beta_{\alpha}$ may remain in these parameters. Moreover, the sig- 


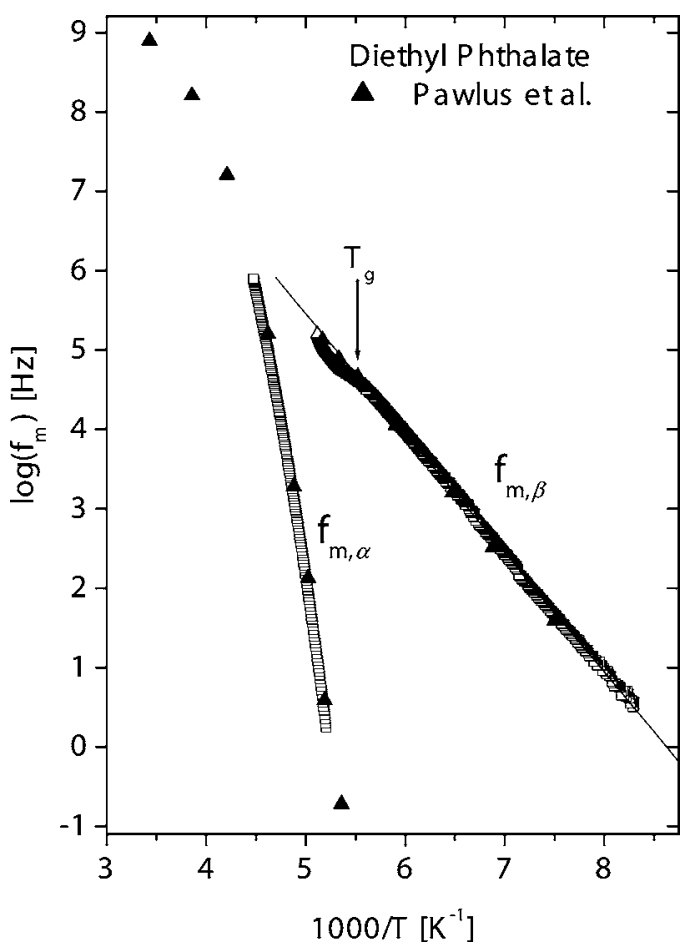

FIG. 4. The relaxation rates for the $\alpha$ relaxation and $\beta$ relaxation for diethyl phthalate obtained from Eq. (2) are plotted as $\log \left(f_{m}\right)$ against $1000 / T$. The data from Pawlus et al. ${ }^{6}$ are shown for comparison. Over the temperature range $120.4-180 \mathrm{~K}, \log \left(f_{m, \beta}\right)$ follows the Arrhenius equation $f_{m, \beta}$ $=10^{13} \exp (-28800 / R T) \cdot \log \left(f_{m, \beta}\right)$ data above $180 \mathrm{~K}$ are indicated by triangles.

nificance of the distribution parameters, $\beta_{\alpha}$ and $\alpha_{\beta}$, is not yet understood. Therefore, we can reliably deduce only that the activation energy of the $\beta$ relaxation is higher for the ultraviscous liquid than for the glassy state, but not exactly how much higher.

Fujima et $\mathrm{al}^{34}$ have observed a similar feature in the plot for D-sorbitol. They have shown it as a plot of the symmetric broadening parameter for the $\beta$ relaxation against $1 / T$ in Fig. 4(b) of Ref. 34. Moreover, they have also observed a slight decrease in the slope of $f_{m, \beta}$ against $1 / T$ plot at $T$ near $T_{g}$ and an increase in the slope at $T>T_{g}$ for D-sorbitol (Fig. 3 in Ref. 34) and only an increase in the slope at $T>T_{g}$ for $m$-fluoroaniline (Fig. 5 in Ref. 34). They have concluded that the $\alpha$ and $\beta$ processes for $\mathrm{D}$-sorbitol and $m$-fluoroaniline do not merge at the Donth temperature in the manner found for other substances. Diethyl phthalate seems to show the same feature as D-sorbitol.

Although a detailed resolution of the spectra for D-sorbitol has supported their findings, ${ }^{34}$ we investigate if the features observed here could be seen as an artifact of local temperature change in the sample as a result of structural relaxation during the course of measurements. A possible local increase in the temperature as a result of structural relaxation at $T<T_{g}$, would raise $f_{m}$ and would therefore increase the slope of the $\log \left(f_{m, \beta}\right)$ against $1 / T$ plot at $T$ near $T_{g}$, but here a decrease is observed in Fig. 4. Furthermore, any local cooling effect as a result of the endothermic recovery of the equilibrium liquid state after the temperature has crossed the equilibrium line would decrease $f_{m}$, and hence decrease the slope of the $\log \left(f_{m, \beta}\right)$ against $1 / T$ plot with increase in $T$. But in contrast, the slope in Fig. 4 increases above $T_{g}$. Therefore, we conclude that the features observed in the $\log \left(f_{m, \beta}\right)$ against $1 / T$ plot and of the $\alpha_{\beta}$ against $T$ plots for diethyl phthalate and other liquids ${ }^{34}$ are either a consequence of the multiparameter fits in which the Cole-Cole distribution of times has been used for the $\beta$ relaxation or else they indicate a structural change in this region. It is also conceivable that these features are an indication of the effect of structural unfreezing on heating at $T$ near $T_{g}$.

\section{DISCUSSION}

\section{A. Structure dependence of the relaxation strength and rate}

There is little doubt that $\Delta \epsilon_{\beta}$ would be greatly affected by the manner of fitting of the data. However, one may argue that part of the change in $\Delta \epsilon_{\beta}$ could be an artifact of local temperature change in the sample as a result of structural relaxation during the course of measurements. The plot of $\Delta \epsilon_{\beta}$ in Fig. 3(A) clearly shows that its magnitude increases slowly as the diethyl phthalate glass is heated from 120 to $150 \mathrm{~K}$. The rate of increase $d \Delta \epsilon_{\beta} / d T$ decreases thereafter until $T$ of $176 \mathrm{~K}$ is reached, becoming almost zero in an irreversible manner. This is a consequence of structural relaxation of the glass and it has been found to be most pronounced for heating the quenched glassy state of 5-methyl-2-hexanol, ${ }^{29}$ less for D-sorbitol, ${ }^{30}$ and somewhat least for chlorobenzene-decalin mixture. ${ }^{1}$ The effect cannot be attributed to local increase of the sample's temperature as a result of heat released because such an increase would raise $\Delta \epsilon_{\beta}$, instead of decreasing it or keeping it constant. Thereafter, $\Delta \epsilon_{\beta}$ in Fig. 4(A) increases slowly in the $T_{g}$ range and it then increases rapidly and reversibly in the equilibrium ultraviscous liquid at $T>T_{g}$, and its high value is independent of time. This increase also cannot be attributed to a possible local cooling that may occur as a result of the endothermic recovery of the equilibrium liquid state after the sample's temperature has crossed the equilibrium line. (See Fig. 12.1 in Ref. 35 for details of the exothermic structural relaxation to a lower energy state at $T<T_{g}$ and endothermic recovery of the equilibrium liquid at $T>T_{g}$, after crossing the equilibrium liquid line.) We conclude that these artifacts, if present, would tend to diminish the features observed here, thus suggesting the possibility that the actual effect may be larger.

The temperature derivative of $\Delta \epsilon_{\beta}$ has a broad discontinuity at $T_{g}$ as has been found for $\Delta \epsilon_{\beta}$ in chlorobenzenedecalin solution, ${ }^{1}$ 5-methyl-2-hexanol, ${ }^{29}$ and D-sorbitol. ${ }^{30}$ The slope of the $\log \left(f_{m, \beta}\right)$ vs $1 / T$ plot has also been found to be more negative below $T_{g}$ for a quenched 5-methyl-2-hexanol ${ }^{29}$ and D-sorbitol ${ }^{30}$ glass-the effect decreasing as $T_{g}$ is approached on heating. Hence, the temperature dependence of the $\beta$-relaxation process mimics the temperature dependence of the enthalpy, entropy, and volume of a glass and ultraviscous liquid.

The greater sensitivity of $\Delta \epsilon_{\beta}$ and $f_{m, \beta}$ of the ultraviscous liquid than in the glassy state of diethyl phthalate seems to be consistent with the observation that the volume, enthalpy, and entropy are more sensitive to temperature at $T$ $>T_{g}$ than at $T<T_{g}$. This would imply that as thermal expan- 
sion of the bulk state becomes higher on crossing $T_{g}$, the population and/or size of the localized regions in an ultraviscous liquid increases, and $\Delta \epsilon_{\beta}$ and $f_{m}$ increases more rapidly. Independent studies by PALS measurement of polypropylene glycol-4000, ${ }^{21}$ cis-trans 1,4 poly-butadiene, ${ }^{21}$ cis 1,4 polyisoprene, ${ }^{21}$ trans 1,4 polychloroprene, ${ }^{21}$ selenium, ${ }^{23}$ glycerol, ${ }^{20,22,24,25}$ and $o$-terphenyl (originally made by Malhotra and Pethrick ${ }^{36}$ and later analyzed by Bartoš et $a l^{21}$ ) have shown a similar elbow-shaped plots of the hole volume against the temperature. Moreover, it has been inferred that small cavities of mean equivalent radius of 2.4 to $4.8 \AA$ occur in ultraviscous liquid glyoerol at $250-275 \mathrm{~K}$, and their limiting size is temperature dependent more at $T>T_{g}$ than at $T<T_{g}$. Although these results do not directly refer to the $\beta$-relaxation process, it seems that the rate at which the size of the hole volume increased with $T$ may also determine the rate at which $\Delta \epsilon_{\beta}$ and $f_{m, \beta}$ increase with $T$. Since changes in the hole volume, and the macroscopic $V, H$, and $S$ are a reflection of changes in the structure of an ultraviscous liquid, we conclude that there is a structural contribution to the $\beta$ relaxation which becomes kinetically frozen in on cooling through $T_{g}$. Therefore, the thermodynamic state functions of ultraviscous liquids and glasses would contain contributions from the vibrational and configurational partition functions for both the $\alpha$ - and the $\beta$-relaxation processes. At temperatures above the ( $\alpha$ - and $\beta$-relaxation processes merging) Donth temperature, where the $\alpha$-relaxation process is absent, the partition function at those temperatures would be only due to the $\beta$ relaxation.

\section{B. Entropy and $\beta$ relaxation}

Simha et al. ${ }^{37}$ had concluded that the thermal expansion coefficient of some polymer glasses has a contribution from the availability of configurations from $\beta$-relaxation motions and Goldstein ${ }^{13,14}$ had concluded that the heat capacity $C_{p}$ and entropy $S$ of a glass may also have a significant contribution from this relaxation. By using the calorimetric data from Chang et al., ${ }^{3}$ Goldstein ${ }^{13,14}$ had shown that entropy $S_{\text {exc }}$ of annealed diethyl phthalate glass ${ }^{3}$ over the entropy of its crystal phase is $22.25 \mathrm{~J} / \mathrm{mol} \mathrm{K}$ at (adiabatic calorimetric) $T_{g}$ of $178 \mathrm{~K}$. The $0 \mathrm{~K}$ entropy of the annealed glass is $20.17 \mathrm{~J} / \mathrm{mol} \mathrm{K}$ and thus the increase in $S_{\text {exc }}$ from $0 \mathrm{~K}$ to $T_{g}$ for the annealed glass is $2.1 \mathrm{~J} / \mathrm{mol} \mathrm{K}$. For a diethyl phthalate glass formed by quenching, ${ }^{3}$ the increase in $S_{\text {exc }}$ over the $0 \mathrm{~K}$ value is $3.64 \mathrm{~J} / \mathrm{mol} \mathrm{K}$ higher than the annealed glass at $T$ near $T_{g}$ of $178 \mathrm{~K}$ (Table I of Ref. 14) and at $T=180.8 \mathrm{~K}$, it is $1.7 \mathrm{~J} / \mathrm{mol} \mathrm{K}$. The increase in the entropy of the quenched glass over the annealed glass is due to several factors: (i) the entropy difference from greater anharmonicity of vibrational displacement, (ii) the difference in the vibrational frequencies of the glass and crystal phases, (iii) the change in the number of configurational states that involve only the $\beta$-relaxation motions, and (iv) the loss in the annealed glass of available configurations from the unfrozen modes of molecular motions that show up as the high-frequency tail of the $\alpha$-relaxation time distribution in the dielectric spectra. ${ }^{17}$ The

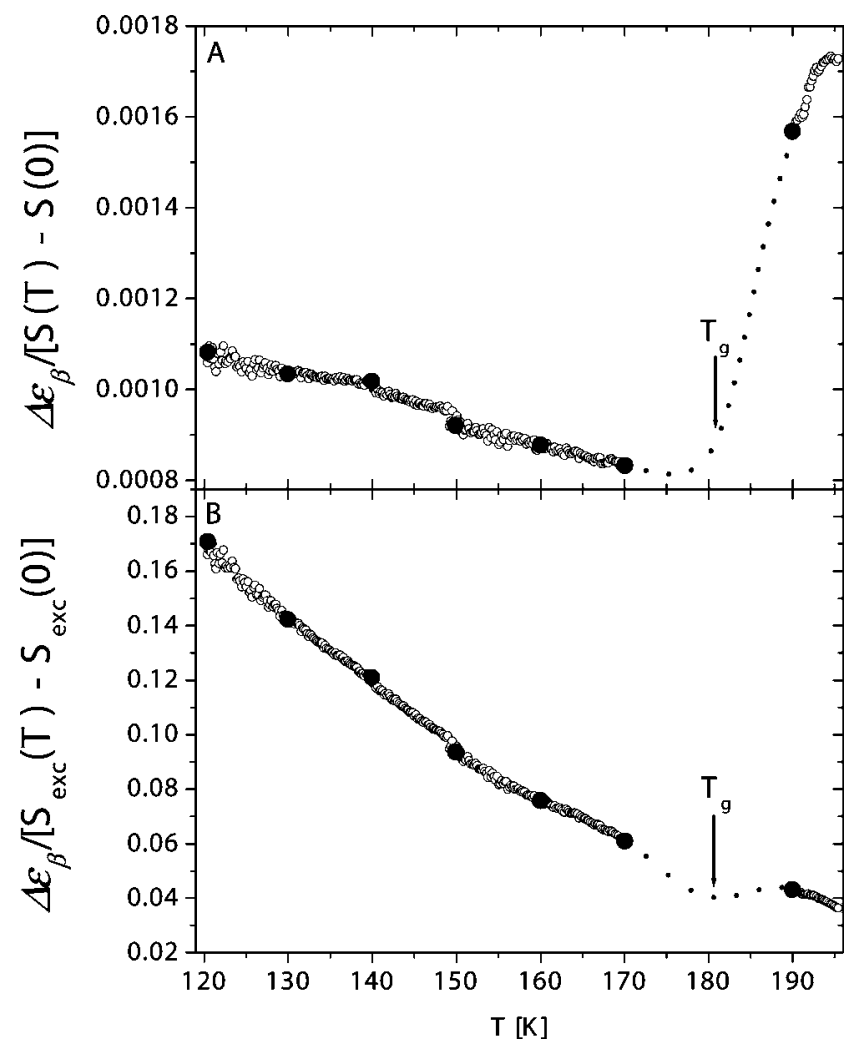

FIG. 5. (A) The ratio $\Delta \epsilon_{\beta} /[S(T)-S(0)]$ for quenched diethyl phthalate is plotted against the temperature. (B) The ratio $\Delta \epsilon_{\beta} /\left[S_{\text {exc }}(T)-S_{\text {exc }}(0)\right]$ is plotted against the temperature. The entropies at each temperature were found by interpolating entropy data of the "quenched glass" taken from Table VI of Chang et al. ${ }^{3}$ and using the $S_{\text {exc }}(0)$ value given in that paper. The values of the ratios at the actual data points of Chang $e t$ al. are indicated by filled circles. The interpolation between 170 and $190 \mathrm{~K}$ when there are no $S_{\text {exc }}(T)$ or $S(T)$ data is shown as a dotted line.

last of these is observed as a relatively rapid decrease in $S_{\text {exc }}$ and $C_{p \text { exc }}$ on cooling a glass to a temperature a few degrees below its $T_{g}$.

To examine the relation between $\Delta \epsilon_{\beta}$ and the entropy, we have plotted the ratio $\Delta \epsilon_{\beta} /[S(T)-S(0)]$ against $T$ in Fig. $5(\mathrm{~A})$, for the quenched glass using the diethyl phthalate entropy data for a "quenched glass" reported by Chang et al. ${ }^{3}$ The ratio $\Delta \epsilon_{\beta} /\left[S_{\text {exc }}(T)-S_{\text {exc }}(0)\right]$ is also plotted against $T$ in Fig. 5(B), where $S_{\text {exc }}(T)$ is the excess entropy at $T \mathrm{~K}$ and $S_{\text {exc }}(0)$ is the excess entropy at $0 \mathrm{~K}$. Since the available entropy data are at 5 or $10 \mathrm{~K}$ temperature intervals, with a $20 \mathrm{~K}$ gap in the glass and ultraviscous liquid entropy data over the time- dependent, glass-softening or $T_{g}$ range, the $S(T)$ and $S_{\text {exc }}(T)$ values for the temperature at which $\Delta \epsilon_{\beta}$ was measured were interpolated from Chang's data by a cubicspline method. Interpolating $S_{\mathrm{exc}}(T)$ in this way gave the same result as interpolating $S(T)$ and separately interpolating the crystal's entropy (which is smooth with no $20 \mathrm{~K}$ gap around $T_{g}$ ) to find $S_{\text {exc }}(T)$. Since there are no entropy data between 170 and $190 \mathrm{~K}$, interpolation in this region, which especially in the case of $S_{\mathrm{exc}}(T)$ may be less reliable, has been shown as a dotted line. The plot of $\Delta \epsilon_{\beta} /[S(T)-S(0)]$ against $T$ in Fig. 5(A) shows that on heating from $120 \mathrm{~K}$, $\Delta \epsilon_{\beta}$ increases more slowly than $[S(T)-S(0)]$, and the plot has a negative slope. This slope approaches zero at $\sim 176 \mathrm{~K}$, 

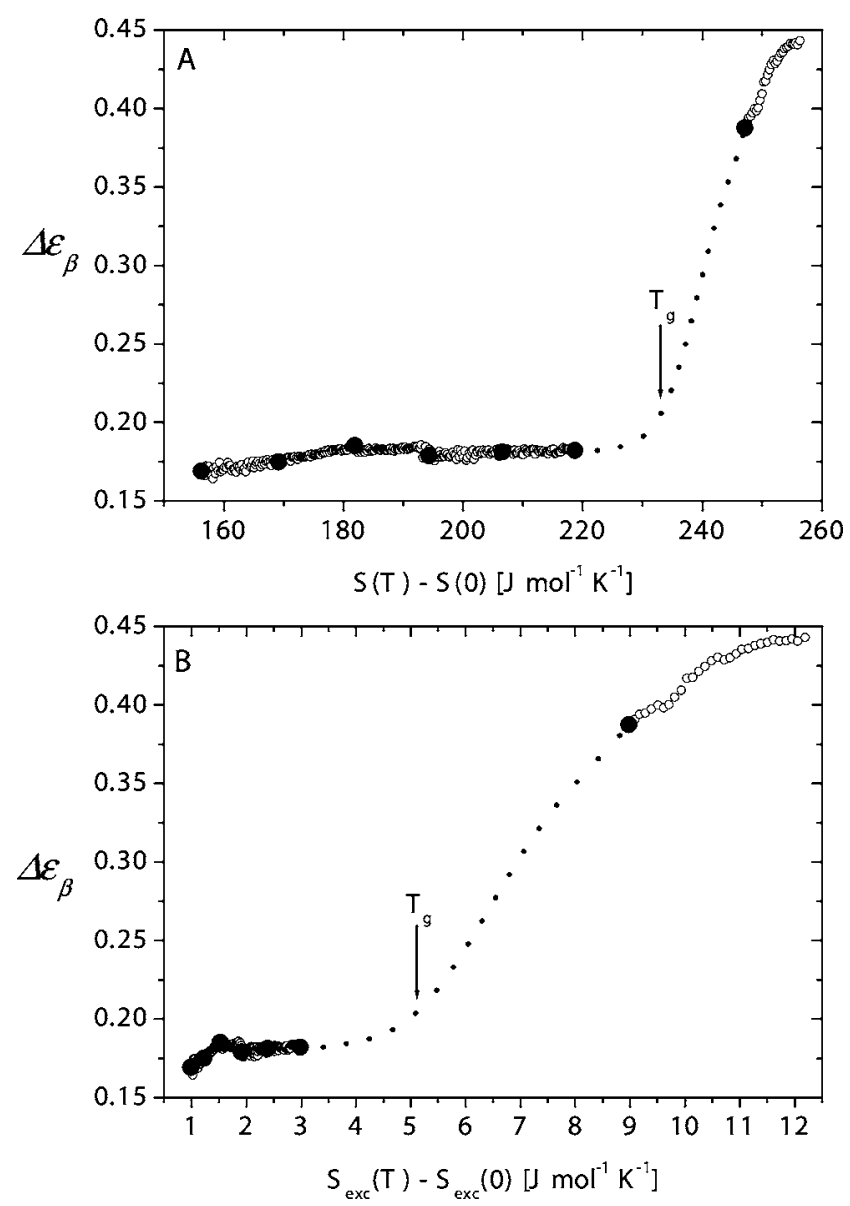

FIG. 6. (A) $\Delta \epsilon_{\beta}$ for quenched diethyl phthalate is plotted against $[S(T)$ $-S(0)]$. (B) $\Delta \epsilon_{\beta}$ is plotted $\left[S_{\text {exc }}(T)-S_{\text {exc }}(0)\right]$. The entropy values at each temperature were found by interpolating entropy data for the quenched glass taken from Table VI of Chang et $\mathrm{al}^{3}$ and using the value of $S_{\text {exc }}(0)$ given in that paper. The values of $\Delta \epsilon_{\beta}$ corresponding to the entropy data points of Chang et al. are indicated by filled circles. The interpolation between 170 and $190 \mathrm{~K}$ when there are no $S_{\text {exc }}(T)$ or $S(T)$ data is shown as a dotted line.

and thereafter $\Delta \epsilon_{\beta}$ increases more rapidly than $[S(T)-S(0)]$, and the plot of $\Delta \epsilon_{\beta} /[S(T)-S(0)]$ against $T$ has a positive slope.

The plot of $\Delta \epsilon_{\beta} /\left[S_{\text {exc }}(T)-S_{\text {exc }}(0)\right]$ against $T$ in Fig. 5(B) decreases continuously as $T$ increases. Interpolation of the data from $170-190 \mathrm{~K}$ suggests that there is a shallow dip from $\sim 176-190 \mathrm{~K}$, where the ratio decreases more sharply, with a minimum around $T_{g} . \Delta \epsilon_{\beta} /\left[S_{\text {exc }}(T)-S_{\text {exc }}(0)\right]$ then continues to decrease at $T>T_{g}$. Within the experimental uncertainty, the similarity in the shape of the plot indicates that $\Delta \epsilon_{\beta}$ and $\left[S_{\text {exc }}(T)-S_{\text {exc }}(0)\right]$ change together in the same qualitative manner and the increase in $\Delta \epsilon_{\beta}$ is less than that in $\left[S_{\text {exc }}(T)-S_{\text {exc }}(0)\right]$ in the $T_{g}$ range. Thus there seems to be a qualitative relation between the entropy and $\Delta \epsilon_{\beta}$ of diethyl phthalate glass and viscous liquid. This is more clearly seen from the plots in Figs. 6(A) and 6(B), where $\Delta \epsilon_{\beta}$ is plotted against $[S(T)-S(0)]$ and against $\left[S_{\text {exc }}(T)-S_{\text {exc }}(0)\right]$, respectively. This establishes a parallel between the entropy and $\Delta \epsilon_{\beta}$ of a glass and liquid, and it shows that temperature dependence of the population of molecules whose motion contributes to $\Delta \epsilon_{\beta}$ is effected by the kinetic freezing (or unfreezing) of the ultraviscous liquid. It also means that: (i) configurational entropy of a liquid has a role in determining the magnitude of $\Delta \epsilon_{\beta}$, (ii) the plot of its temperature derivative $\left(d \Delta \epsilon_{\beta} / d T\right)$ against $T$ would appear like the plot of the heat capacity against $T$, (iii) the glass-softening temperature range can be determined from the knowledge of $\Delta \epsilon_{\beta}$, and (iv) the more rapid increase in the hole volume in the ultraviscous liquid than in the glassy state seems parallel to the increase in the population of molecules contributing to the $\beta$ relaxation. These features seem to underscore the general notion that $\beta$ relaxation is associated with local-density fluctuations that kinetically freeze when a liquid vitrifies, creating regions of low density in the glass structure.

\section{Molecular origin of the $\boldsymbol{\beta}$ relaxation}

There are currently two views on the molecular origin of the $\beta$ relaxation. In one view, thermally activated motions of only a small fraction of the total number of molecules contribute to the $\beta$ relaxation. ${ }^{4,14,16-18,38}$ Such molecules or molecular groups are at the sites of low density in a kinetically frozen disordered structure. This would be expected on the basis of freezing in of the density fluctuations. In the second view, which is based on the NMR observations, $\beta$ relaxation involves thermally activated, small-angle motion of all molecules or essentially all molecules in the disordered structure. ${ }^{39,40}$ It is only after this motion has occurred that the $\alpha$ relaxation, which involves large-angle motion of the same molecules begins. Johari ${ }^{41}$ had given several arguments against the small-angle motions of all molecules as a mechanism for the process. One of these arguments was ${ }^{41}$ that if all molecules were to contribute to $\beta$ relaxation, the entropy due to the number of available configurations would be at least $R \ln 2$ or $5.76 \mathrm{~J} / \mathrm{mol} \mathrm{K}$, given that there are only two sites available for a molecule to diffuse and there is no redundancy of configurations in a positionally disordered structure. This means that if all the entropy were lost on cooling a glass from $T_{g}$ to near $0 \mathrm{~K}$, the decrease should be at least $5.76 \mathrm{~J} / \mathrm{mol} \mathrm{K}$. If the $\beta$-relaxation process itself was to become kinetically frozen on cooling to $0 \mathrm{~K}$, the decrease would be less than $5.76 \mathrm{~J} / \mathrm{mol} \mathrm{K}$, but the residual entropy would then include a substantial amount of the entropy due to disorder from frozen-in configurations involving smallangle motions.

By interpolating the data of Chang et al. ${ }^{3}$ for quenched glass by the method described above, we determined the excess entropy of the quenched glass over the crystal phase less the value at $0 \mathrm{~K},\left[S_{\mathrm{exc}}(T)-S_{\mathrm{exc}}(0)\right]$. [It is $2.92 \mathrm{~J} / \mathrm{mol} \mathrm{K}$ at $178 \mathrm{~K}$ (Ref. 14)]. It is determined here as $5.13 \mathrm{~J} / \mathrm{mol} \mathrm{K}$ at $180.8 \mathrm{~K}$, which is still in the glass-softening range. The quantity $\left[S_{\text {exc }}(T)-S_{\text {exc }}(0)\right]$ includes the contribution to the entropy from greater anharmonicity of vibrational displacement, difference in the vibrational frequencies of the glass and crystal phase and the loss of configurations from the unfrozen modes of motion that show up as the highfrequency tail of the $\alpha$-relaxation distribution in dielectric and mechanical spectra, and relatively rapidly decreasing $S_{\text {exc }}$ and $C_{p \text {,exc }}$ on cooling a glass to a temperature a few degrees below its $T_{g}{ }^{17}$ The entropy associated with the $\beta$-relaxation configurations would therefore be much less than $2.92 \mathrm{~J} / \mathrm{mol} \mathrm{K}$ at $178 \mathrm{~K}$ and $5.1 \mathrm{~J} / \mathrm{mol} \mathrm{K}$ at $180.8 \mathrm{~K}$. 
Since the minimum entropy for the $\beta$-relaxation process from motion of all molecules is at least $5.76 \mathrm{~J} / \mathrm{mol} \mathrm{K}$ ( $=R \ln 2$ for a minimum of only two distinct configurations), and the measured entropy attributable to $\beta$ relaxation is much less than this value, we conclude that the entropy and heat capacity data of diethyl phthalate glass do not support the view that $\beta$ relaxation involves small-angle motions of all molecules in a disordered structure. This is independent of the Ngai and Paluch's ${ }^{7}$ conclusion that the relaxation is not the Johari-Goldstein relaxation.

It is also worth noting that small-angle displacements of all molecules, angular or translational, may not be valid as a mechanism for $\beta$ relaxation, generally. To elaborate, structural relaxation of a glass has been shown to decrease the height of the $\beta$-relaxation peak, ${ }^{16,29}$ the $\Delta \epsilon_{\beta},{ }^{16,29}$ and the entropy. Now, if all molecules were to contribute to $\beta$ relaxation, this entropy will not change on structural relaxation unless some configurations became unavailable. Alternatively, the entropy would decrease to a minimum value of $R \ln 2$ and remain so as long as all molecules contributed to it. After this value has been reached, thermodynamics and molecular dynamics of this process would become unrelated. Also, if this mechanism was to be generally applicable to all glasses, glassy metals, which are composed of atoms would not show a $\beta$ relaxation, because atomic motions can only be translational, and any translational motion, howsoever small, of all molecules in a glass structure would lead to an irrecoverable deformation of the glass, as pointed out earlier. ${ }^{41}$ But $\beta$ relaxation does not lead to an irrecoverable deformation. Finally, recent PALS studies of molecular and polymeric glasses have indicated the presence of holes of $\sim 1.7-2.1 \AA$ average radius at $T<T_{g}$, as calculated for glycerol from the volume plot in Fig. 2 in Ref. 20 and for polybutadiene from Fig. 3 in Ref. 21 whose size increases with the temperature, and the plot of the hole volume against $T$ of different polymers and molecular materials show a qualitatively similar elbow shaped, ${ }^{20-25}$ as the plot of $\Delta \epsilon_{\beta}$ observed here. These issues need to be resolved before interpreting the NMR studies of $\beta$ relaxation in terms of small-angle motions of all molecules in the structure. The free volume and the entropy theories of glass formation have made no provision for the occurrence of molecular motions in the glassy and ultraviscous liquid state, mainly because at that time these motions were recognized as extrinsic to the structure of the glass. The phenomenologies of the energy landscape model, of the coupling model, and of the various treatments of the asymmetric potential well models also do not consider the molecular or structural origin of the $\beta$ relaxation.

\section{CONCLUSION}

The orientation polarization associated with the $\beta$-relaxation process increases slowly with increase in temperature in the glassy state and rapidly in the ultraviscous liquid state. This is remarkably similar to the changes that are observed for the volume, enthalpy, and entropy of a supercooled liquid and glass and ultraviscous liquid, which means that $T_{g}$ can be equally well characterized by the strength of the $\beta$-relaxation process. The activation energy of the $\beta$-relaxation rate seems to decrease when an ultraviscous liquid becomes a glass on cooling. Therefore it seems that the activation energy of the $\beta$ relaxation in the ultraviscous liquid has two components, one is structure dependent and the second is not. In the glassy state only the second is observed. We expect that a similar decrease will be found in the volume of activation at the glass-transition pressure, i.e., the pressure at which a liquid would vitrify when compressed at fixed temperature. Thus the glass-transition range of a liquid may be equally well defined from measurements of the dielectric behavior of its $\beta$-relaxation process.

The strength of $\beta$ relaxation changes as the net entropy changes, but the extents of their respective change vary with the temperature. When the change in the $\beta$ relaxation's strength is compared with the calorimetric entropy (the entropy above the $0 \mathrm{~K}$ value) the extents of their respective change with the temperature are closely similar. Configurational entropy of a liquid has a role in determining the magnitude of $\Delta \epsilon_{\beta}$. Moreover, the glass-softening temperature range can be determined from the knowledge of temperature dependence of $\Delta \epsilon_{\beta}$. When combined with the known observations that the hole volume increases more rapidly in the ultraviscous liquid than in the glassy state, the results indicate that $\beta$ relaxation is associated with local-density fluctuations that kinetically freeze when a liquid vitrifies. Hence, low-density regions or holes become frozen in the glass structure.

The features of the $\beta$-relaxation process seems to be consistent with the view that fast molecular motions are localized in the structures of a glass and ultraviscous liquid, and may not be associated with the small-angle motions of all molecules in their structures.

\section{ACKNOWLEDGMENTS}

The authors are grateful to INTEL Ireland Ltd. for funding the scholarship of one of the authors (G.P.). Another author (G.P.J.) would like to thank Trinity College for their hospitality during his stay for this study funded by the SFI overheads. We are grateful to Professor J. Bartoš for informing us that the hole volume temperature plots they had obtained resemble our plots for $\Delta \epsilon_{\beta}$ for chlorobenzene-cis decalin solution in Ref. 1. He kindly sent us papers cited in Refs. 19-25, of which we were unaware. A reading of those papers has resulted in our discussion of the implications of the PALS studies. Some of the entropy consideration here may be useful for combining the hole volume variation, which they have related to the free volume. Also a recent paper by Ichitsubo et $a l .^{42}$ has described a microstructural model for a glass, consisting of a mixture of weakly bonded regions (islands of mobility) and strongly-bonded regions.

\footnotetext{
${ }^{1}$ G. P. Johari, G. Power, and J. K. Vij, J. Chem. Phys. 116, 5908 (2002). ${ }^{2}$ S. S. Chang and A. B. Bestul, J. Chem. Phys. 56, 503 (1972).

${ }^{3}$ S. S. Chang, J. A. Horman, and A. B. Bestul, J. Res. Natl. Bur. Stand., Sect. A 71A, 293 (1967)

${ }^{4}$ G. P. Johari and M. Goldstein, J. Chem. Phys. 53, 2372 (1970).

${ }^{5}$ G. P. Johari and M. Goldstein, J. Chem. Phys. 55, 4245 (1971).

${ }^{6}$ S. Pawlus, M. Paluch, M. Sekula, K. L. Ngai, S. J. Rzoska, and J. Ziolo, Phys. Rev. E 68, 021503 (2003).

${ }^{7}$ K. L. Ngai and M. Paluch, J. Chem. Phys. 120, 857 (2004).
} 
${ }^{8}$ H. Fujimori and M. Oguni, J. Chem. Thermodyn. 26, 367 (1994).

${ }^{9}$ H. Fujimori and M. Oguni, Solid State Commun. 94, 157 (1995).

${ }^{10}$ H. Fujimori, M. Mizukami, and M. Oguni, J. Non-Cryst. Solids 204, 38 (1996).

${ }^{11}$ S. V. Nemilov and G. P. Johari, Philos. Mag. 83, 3117 (2003); S. V. Nemilov and G. P. Johari, Philos. Mag. 84, 845 (2004).

${ }^{12}$ J. P. Joule, Memr. Manchester Literary Phil. Soc. 3, 292 (1867); The Scientific Papers of J. P. Joule (London Physical Society, London, 1884), Vol. 1, p. 558.

${ }^{13}$ M. Goldstein, Ann. N.Y. Acad. Sci. 279, 68 (1976).

${ }^{14}$ M. Goldstein, J. Chem. Phys. 64, 4767 (1976).

${ }^{15}$ G. P. Johari, Ann. N.Y. Acad. Sci. 279, 102 (1976).

${ }^{16}$ G. P. Johari, Philos. Mag. B 41, 41 (1980).

${ }^{17}$ G. P. Johari, J. Chem. Phys. 112, 7518 (2000).

${ }^{18}$ G. P. Johari, Ann. N.Y. Acad. Sci. 279, 117 (1976).

${ }^{19} \mathrm{~J}$. Bartoš, in Encyclopedia of Analytical Chemistry, edited by R. A. Meyers (Wiley, Chichester, 2000), p. 7968.

${ }^{20}$ J. Bartoš, O. Šauša, J. Krištiak, T. Blochowicz, and E. Rössler, J. Phys.: Condens. Matter 13, 11473 (2001).

${ }^{21}$ J. Bartoš, O. Šauša, P. Bandzuch, J. Zrubcová, and J. Krištiak, J. NonCryst. Solids 307-310, 417 (2002).

${ }^{22}$ J. Bartoš, O. Šauša, D. Račko, J. Krištiak, and J. J. Fontanella, J. NonCryst. Solids 351, 2599 (2002).

${ }^{23}$ J. Bartoš, O. Šauša, P. Pustková, J. Shánělová, J. Krištiak, and J. Málek, J. Non-Cryst. Solids 351, 1082 (2005).

${ }^{24}$ J. T. Bendler, J. J. Fontanella, M. F. Schlesinger, J. Bartoš, O. Šauša, and J. Krištiak, Phys. Rev. E 71, 031508 (2005).
${ }^{25}$ D. Račko, R. Chelli, G. Cardeni, J. Bartoš, and S. Califano, Eur. Phys. J. D 32, 289 (2005).

${ }^{26}$ G. Power, Ph.D. thesis, University of Dublin, Ireland, 2005.

${ }^{27}$ S. Havriliak, Jr. and S. Negami, J. Polym. Sci., Part C: Polym. Symp. 14, 99 (1966).

${ }^{28}$ O. E. Kalinovskaya and J. K. Vij, J. Chem. Phys. 112, 3262 (2000).

${ }^{29}$ G. P. Johari, G. Power, and J. K. Vij, J. Chem. Phys. 117, 1714 (2002).

${ }^{30}$ G. Power, G. P. Johari, and J. K. Vij, J. Chem. Phys. 119, 435 (2003).

${ }^{31}$ K. S. Cole and R. H. Cole, J. Chem. Phys. 9, 341 (1941).

${ }^{32}$ D. W. Davidson and R. H. Cole, J. Chem. Phys. 19, 1484 (1951).

${ }^{33}$ C. Hansen, F. Stickel, T. Berger, R. Richert, and E. W. Fischer, J. Chem. Phys. 107, 1086 (1997).

${ }^{34}$ T. Fujima, H. Frusawa, and K. Ito, Phys. Rev. E 66, 031503 (2002).

${ }^{35}$ G. W. Scherer, Relaxations in Glass and Composites (Wiley, New York, 1986), Chap. 12.

${ }^{36}$ B. D. Malhotra and R. A. Pethrick, J. Chem. Soc., Faraday Trans. 2 78, 297 (1982).

${ }^{37}$ R. Simha, J. M. Roe, and V. S. Nanda, J. Appl. Phys. 43, 4312 (1972).

${ }^{38}$ G. P. Johari, J. Chem. Phys. 77, 4619 (1982), and references therein.

${ }^{39}$ M. Vogel, C. Tschirwitz, G. Schnieder, C. Koplin, P. Medick, and E. Rössler, J. Non-Cryst. Solids 307-310, 326 (2002).

${ }^{40}$ A. Döß, M. Paluch, H. Sillescu, and G. Hinze, J. Chem. Phys. 117, 6582 (2002).

${ }^{41}$ G. P. Johari, J. Non-Cryst. Solids 307-310, 317 (2002).

${ }^{42}$ T. Ichitsubo, E. Matasubara, T. Yamamoto, H. S. Chen, N. Nishiyama, J. Saida, and K. Anazawa, Phys. Rev. Lett. 95, 245501 (2005). 